\title{
Emmanuel Carrère. Le point de vue de l'adversaire, éds. Christophe REIG, Alain ROMESTAING et Alain SCHAFFNER
}

\author{
Giovanni Tallarico
}

\section{(2) OpenEdition}

\section{Journals}

\section{Edizione digitale}

URL: https://journals.openedition.org/studifrancesi/12183

DOI: 10.4000/studifrancesi. 12183

ISSN: 2427-5856

\section{Editore}

Rosenberg \& Sellier

\section{Edizione cartacea}

Data di pubblicazione: 1 avril 2018

Paginazione: 171-172

ISSN: 0039-2944

\section{Notizia bibliografica digitale}

Giovanni Tallarico, «Emmanuel Carrère. Le point de vue de l'adversaire, éds. Christophe REIG, Alain ROMESTAING et Alain schafFNeR», Studi Francesi [Online], 184 (LXII | I) | 2018, online dal 03 juillet 2018, consultato il 17 novembre 2021. URL: http://journals.openedition.org/studifrancesi/12183 ; DOI: https://doi.org/10.4000/studifrancesi. 12183

Questo documento è stato generato automaticamente il 17 novembre 2021.

\section{(†) $\odot$

Studi Francesi è distribuita con Licenza Creative Commons Attribuzione - Non commerciale - Non opere derivate 4.0 Internazionale. 


\title{
Emmanuel Carrère. Le point de vue de l'adversaire, éds. Christophe REIG, Alain ROMESTAING et Alain SCHAFFNER
}

\author{
Giovanni Tallarico
}

\section{NOTIZIA}

Emmanuel Carrère. Le point de vue de l'adversaire, éds. Christophe REIG, Alain ROMESTAING et Alain SCHAFFNER, Paris, Presses Sorbonne Nouvelle, 2017, 170 pp.

1 Fedeli al titolo del volume, gli studi qui raccolti tematizzano un aspetto centrale dell'opera di Emmanuel Carrère: l'alterità. Il concetto riassume una duplice accezione per l'autore: l'Altro è in primo luogo altro da sé, l'oppositore con cui può nascere una relazione "agonistica" (come in L'adversaire), ma naturalmente anche l'avversario interno, capace di suscitare pulsioni autodistruttive e dubbi angosciosi e paralizzanti, tanto a livello esistenziale quanto nel processo creativo.

2 Christophe REIG, in (Morceaux de) "Bravoure" - entre romanesque en lambeaux et vol du vampire (pp. 17-29), mostra come in uno dei primi romanzi di Carrère (Bravoure, 1984) emergano in nuce temi e caratteri che si ritroveranno nella produzione successiva dell'autore, in particolare la messa in scena riflessiva del processo creativo dello scrittore, la tendenza all'ibridazione dei generi e un gusto pronunciato per la forma autobiografica e la documentazione. Bravoure ha come modello dichiarato Frankenstein di Mary Shelley, che dapprima imita in tono serio per poi virare al ludico in un gioco ipertestuale e polifonico dove, in definitiva, il mostro è la scrittura, «ce golem de papier» nelle parole di Reig.

3 Anaëlle TABOUL, in La folie en question(s) dans "La moustache" d'Emmanuel Carrère (pp. 31-50), esplora il baratro della follia in uno dei testi più inquieti di Carrère, La moustache (1986). A partire da un evento del tutto banale, il romanzo illustra «une plongée dans l'abîme terrifiant de l'aliénation» (p. 31), che si manifesta nella perdita di sé e nella 
frattura con il mondo circostante. Privato della propria identità, del proprio passato e perfino della propria immagine, il protagonista è vittima di un delirio paranoico che ha come unico esito possibile la fuga e infine il suicidio. Anche il lettore è esposto a un'alienazione simile, impossibilitato com'è a determinare la veridicità degli eventi e in preda a un'incertezza radicale e destabilizzante.

4 Il contributo di Dominique RABATÉ (Est-ce que dire c'est faire? Écrire pour «Faire effraction dans le réel» chez Emmanuel Carrère, pp. 51-61) mette a fuoco l'implacabilità del reale (nel senso lacaniano del termine) nell'opera di Carrère, al cui interno L'adversaire costituisce senza dubbio il livre pivot. Se i primi romanzi tematizzano la finzione come esplorazione angosciosa dei confini tra realtà e immaginazione, i testi della seconda fase mettono in evidenza l'"effrazione" del reale, spesso tragica e violenta, nella vita delle persone. La scrittura performativa messa in atto in Un roman russe è un tentativo, per quanto ambiguo e votato al fallimento, di disinnescare questa minaccia, mentre D'autres vies que la mienne mostra la presa di coscienza della singolarità irriducibile dell'Altro e i suoi effetti catartici sull'autore.

In S'écrire pour s'écrier: une délivrance? (pp. 63-73), Sara BoNOMO si concentra su un trittico di opere dove il passaggio alla prima persona della narrazione è il segno di una svolta radicale nella produzione dell'autore: a partire da L'adversaire, Carrère metterà infatti a nudo se stesso in un gioco di specchi con i personaggi e le loro vite reali. Se Un roman russe è il romanzo delle ossessioni personali dell'autore e dei tentativi per affrancarsene, D'autres vies que la mienne, nel suo slancio verso il mondo e verso gli altri, si colloca sotto il segno della sympatheia.

6 Émilie BRIÈRE, in D'autres vies par la vôtre. Une lecture offerte à Emmanuel Carrère (pp. 75-85), sottolinea la coerenza stilistica e narrativa che contraddistingue due testi antitetici dal punto di vista tematico, ossia L'adversaire e D'autres vies que la mienne. Tale coerenza consiste nell'adozione della prima persona nella narrazione e nella centralità dell'istanza narrativa rispetto al mondo esterno: ciò permette un'attenta esplorazione del sé del narratore-autore e, al tempo stesso, rende possibile raccontare le vite degli altri "tramite" quella di Emmanuel Carrère.

7 Louise LOURDOU, in L'auteur et son double: "Je suis vivant et vous êtes morts", "L'adversaire", "Limonov" (pp. 87-98), indaga le intersezioni tra biografia e autobiografia in tre opere di Carrère. I personaggi dei tre libri, Philip K. Dick, Jean-Claude Romand e Édouard Limonov, suscitano, benché in gradazioni diverse, una certa empatia nell'autore, che si identifica in questi "doppi", mettendo in scena sé stesso in un chiaro esempio di autofiction.

8 Nel saggio Fonctions de l'éloge dans "Limonov" (pp. 99-113), Sylvaine leComte DAUTHUILLE evidenzia il ruolo cruciale che riveste l'elogio in Limonov. Le testimonianze di alcuni personaggi "iconici", tra cui Anna Politkovskaja e Jean Rolin, permettono a Carrère di dissipare i dubbi che nutre riguardo alla moralità dell'anti-eroe Édouard Limonov. L'autore può così acquisire una postura etica rispetto alla propria narrazione e, al tempo stesso, adottare un punto di vista sul mondo che contribuisce a definire la propria identità, spesso minacciata da gravi incertezze ed esitazioni.

9 "Un roman russe" est-il un roman russe? (pp. 115-124), si chiede Nathalie FROLOFF: a una prima lettura, il titolo dell'opera in questione pare infatti paradossale, in quanto l'elemento finzionale è subordinato a un'evidente vocazione autobiografica. Malgrado l'apparente frammentazione e i sottotesti di cui è composta, l'opera possiede comunque, a suo avviso, una forte unità narrativa. Dal punto di vista linguistico, il 
russo è per Carrère l'idioma dell'infanzia e rappresenta una geografia segreta, un luogo mitico intessuto di stereotipi. In secondo luogo, Un roman russe è "russo" poiché si iscrive in continuità con i grandi autori russi dell'Ottocento. Infine, il libro è un'indagine sul valore performativo della scrittura: il tentativo di rendere finzionali personaggi autentici permette di far emergere il romanzesco che agisce nella vita reale, sotto forma di amori infelici o eventi inattesi e straordinari.

10 Alain Romenstaing, Demeurer en l'indécidable (pp. 125-137) osserva come tutta l'opera di Carrère sia contrassegnata dal tema del passaggio e dall'idea di una soglia, più o meno varcabile, che separa da un'esperienza potenzialmente dirompente. Basti pensare al Royaume, resoconto di una "conversione", nella doppia accezione del termine. L'esplorazione del punto di vista altrui permette di intravvedere l'Altro in noi, quella parte di sé che ognuno respinge poiché scardina le proprie certezze impedendo un giudizio netto sulle cose del mondo, che diventano allora letteralmente "indecidibili".

11 Il volume si chiude con una testimonianza dello scrittore Pierre PACHET e con tre interviste, brevi ma significative: a Pachet stesso (scomparso nel 2016 e a cui è dedicata la miscellanea), a CARRÈRE e al suo editore storico, Paul OTCHAKOVSKY-LAURENS (P.O.L.). 